\title{
Special issue on machine learning for robotics
}

\author{
Wei Wei ${ }^{1}$. Jinsong $\mathrm{Wu}^{2} \cdot$ Chunsheng $\mathrm{Zhu}^{3}$
}

Published online: 25 September 2020

(c) Springer-Verlag GmbH Germany, part of Springer Nature 2020

At present, robotics behaves and moves like human, the next step in robotics is towards enhancing robots to think like human and make instantaneous decisions without any human interventions. In order to accomplish this, machine learning algorithms as one important part of artificial intelligence technologies are being introduced into robotics.

This special issue on "Machine Learning for Robotics" provides an ideal platform for researchers and technical experts to share their novel works and analysis pertaining to the field of machine learning and its application in robotics. After a strict peer review, 21 papers are selected for publication in this special issue. Details of these selected papers are as follows.

The paper entitled "Kinematics Model Identification and Motion Control of Robot Based on Fast Learning Neural Network" provided by Xuehong Sun introduces a new learning neural network structure, called fast learning neural network. Results show that it is appropriate to identify the kinematics model and control the motion of the robot based on fast learning neural network.

The paper entitled "Robot Algorithm Based on Neural Network and Intelligent Predictive Control" provided by Yini Wang proposes a novel intelligent predictive control scheme that uses a neural network intelligent predictive controller to control the force/position of the robot. The robustness and rapidity of robots are improved, and good control accuracy and control effects are achieved.

The paper entitled "Error Recognition of Robot Kinematics Parameters Based on Genetic Algorithms" provided by Ying Yan constructs the error model of 6-DOF parallel

\section{Wei Wei}

weiwei@xaut.edu.cn

1 School of Computer Science and Engineering, Xi' an University of Technology, Xi' an 710048, China

2 Department of Electrical Engineering, Universidad de Chile, 833-0072 Santiago, Chile

3 Department of Electrical and Computer Engineering, The University of British Columbia, Vancouver, BC V6T 1Z4, Canada manipulator based on genetic algorithms. The simulation results show that the proposed genetic algorithm-based robot kinematics parameter error identification algorithm has obvious advantages.

The paper entitled "Path Planning and Control of Soccer Robot Based on Genetic Algorithm" provided by Xuanang Chen, et al. proposes a genetic algorithm for the path planning algorithm of a soccer robot, which enables the robot to find a relatively short path from the point to the target.

The paper entitled "Fuzzy Obstacle Avoidance Optimization of Soccer Robot Based on an Improved Genetic Algorithm" provided by Deping Chen studies the method of automatic extraction and optimization of fuzzy rules for fuzzy path planner with the help of an improved genetic algorithm.

The paper entitled "Inverse Kinematics Solution of Robotics Based on Neural Network Algorithms" provided by Ruihua Gao proposes a robotics inverse solution algorithm based on improved BP (back propagation) neural network. The simulation results show that the proposed algorithm in this paper has obvious advantages in solving the kinematics inverse problem of six-degree-of-freedom robotics compared with the traditional inverse solution algorithm.

The paper entitled "Bifurcation of Modified HR Neural Model under Direct Current" provided by Kaijun Wu et al. studies bifurcation of modified HR neural model, and discusses dynamic characteristics of the model. In future it can be applied to study dynamics of robots as a new method.

The paper entitled "Intelligent Automation of Dental Material Analysis using Robotic Arm with Jerk Optimized Trajectory" provided by Robertas Damasevicius et al. propose an innovative robotic platform with ABB high precision industrial robotic arm. The problem of robot trajectory planning to achieve smooth and precise trajectory while minimizing jerk is addressed based on NSGA II multi-objective evolutionary algorithm.

The paper entitled "Adaptive Sliding Mode Control of Robot Based on Fuzzy Neural Network" provided by Tianchi Ye et al. designs a better real-time adaptive control framework to realize the robust target tracking of mobile robots. The simulation results show that the trajectory 
tracking ability, anti-disturbance ability and robustness of the mobile robot are improved.

The paper entitled "Adaptive PID Control of Multi-DOF Industrial Robot Based on Neural Network" provided by Jiyue Wang et al. establishes the functional relationship between control error and arrival degree to improve the control quality of industrial robots based on the core idea of fuzzy neural network algorithm. Experimental results show that the trajectory control algorithm based on the proposed trajectory control algorithm is effective.

The paper entitled "Motion Path Planning of Soccer Training Auxiliary Robot Based on Genetic Algorithm in Fixed-point Rotation Environment" provided by Hui Ding proposes a global path planning method based on genetic algorithm in fixed-point rotation environment, which avoids the problem of unsmooth path and large computation.

The paper entitled "Robot Target Localization and Interactive Multi-mode Motion Trajectory Tracking Based on Adaptive Iterative Learning" provided by Xinming Li proposes a method of robot vision localization based on iterative Kalman particle filter, which realizes the global positioning of the robot. The feasibility and effectiveness of the proposed localization method are verified by simulation results.

The paper entitled "Research and Analysis of Factors Affecting Bending Performance of Multi-cavity Flexible Actuator" provided by Tian Hui et al. designs a multi-cavity flexible actuator, which consists of two parts, a main chamber and a base. The mathematical model of flexible actuator was established and simulated by commercial software to explore the effect of the length, the width and the height of the single chamber of the actuator on its bending performance.

The paper entitled "Cloud-Based Robotic System for Crowd Control in Smart Cities using Hybrid Intelligent Generic Algorithm" provided by K. Manikanda Kumaran et al. proposes a cloud-based robotic system for the crowd control system in smart cities using the Hybrid Intelligent Generic Algorithm (HIGA) for smart crowd management. The experimental results show that the proposed cloudbased robotic system achieves powerful computation, storage, energy-efficient and reduce the cost with the help of cloud computing for smart city management.

The paper entitled "Enhanced Humanoid Assisted Human Interaction Model Based on Linear Structural Modeling for Knowledge Representation" provided by S. Periyanayagi et al. proposes Linear Linguistic Advanced Structural Modeling (LLASM)approach to develop Weighted Linguistic Reasoning (WLR) algorithm for the purpose of reasoning and knowledge representation. In this paper human computer interaction faces optimal flow and job characteristics experience using individual computers in the workplace which helps to overcome the issues using LLASM in a variety of organizations with robotic assistance for verbal and nonverbal sequence using humanoid robot.

The paper entitled "Task Assignment Strategy for MultiRobot Based on Improved Grey Wolf Optimizer" provided by Jing $\mathrm{Li}$ et al. proposes a distributed cooperative task allocation strategy based on Improved Grey Wolf Optimizer (IGWO) algorithm for Multi-Robots. Simulation results show that the task allocation strategy is reasonable, the path length is roughly balanced, the planning cost is small, the convergence speed is fast and the stability is good.

The paper entitled "Research on Remote Control Algorithm for Parallel Implicit Domain Robot Patrol Inspection on 3D Unstructured Grid" provided by Tingyang Li et al. proposes a three-dimensional unstructured grid DSMC parallel algorithm for the remote control on the parallel implicit domain robot patrol inspection. The actual operation shows that the system can remotely access the service system and message transmission of the inspection robot in the state of the sub-structure grid.

The paper entitled "Multimodal Visual Image Processing of Mobile Robot in Unstructured Environment Based on Semi-supervised Multimodal Deep Network" provided by Yajia Li studies the related problems of visual image processing of mobile robots in unstructured environments based on a well-designed semi-supervised multimodal deep network (SMMDN). The simulation results prove that SMMDN improves the perception and recognition ability of mobile robots in unstructured environments.

The paper entitled "Three-Dimensional Reconstruction of the Dribble Track of Soccer Robot Based on Heterogeneous Binocular Vision" provided by Chunyu Tong et al. uses the heterogeneous binocular vision technology to analyze the kinematics of the soccer robot in detail. The reliability, practicability and rationality of the designed model are verified.

The paper entitled "Robotic-based Nonlinear Device Fault Detection with Sensor Fault and Limited Capacity for Communication" provided by R. Janarthanan et al. proposes the fault detection filter for wireless sensor network based on a robotic-based nonlinear device. Results prove the designed filters for a total defect detection system are with a robust mean-square asymptotic stability.

The paper entitled "An Intelligent Navigational Strategy for Mobile Robots in Uncertain Environments using Smart Cuckoo Search Algorithm" provided by Prases K. Mohanty presents the implementation of Smart Cuckoo Search (SCS) algorithm for intelligent path planning of mobile robots. Finally the simulation results are validated with KheperaIV mobile robot experiments and it is revealed that proposed algorithm is valid and feasible in the mobile robot path planning problems.

We are extremely grateful to Prof. Vincenzo Loia, the Editor-in-Chief of Journal of Ambient Intelligence and Humanized Computing, for providing the opportunity to 
organize this special issue, and for the constant and prompt support. We also thank all the authors for their valuable contributions to this special issue, and all the volunteer reviewers for their hard work in evaluating all the submissions and their helpful comments that certainly contributed to the quality of the published papers.
Publisher's Note Springer Nature remains neutral with regard to jurisdictional claims in published maps and institutional affiliations. 